\title{
Can a mindfulness-informed intervention reduce aggressive behaviour in people with intellectual disabilities? Protocol for a feasibility study
}

Gemma Maria Griffith ${ }^{1 *}$, Robert Jones², Richard Patrick Hastings ${ }^{3}$, Rebecca S. Crane ${ }^{1}$, Judith Roberts², Jonathan Williams ${ }^{4}$, Lucy Bryning ${ }^{5}$, Zoe Hoare ${ }^{6}$ and Rhiannon Tudor Edwards ${ }^{5}$

\begin{abstract}
Background: Approximately 10-20\% of adults with intellectual disabilities engage in challenging behaviours such as aggression, destructiveness, and self-injury, which are often accompanied by feelings of anger. The inability to manage anger can reduce quality of life. For example, aggression is a strong predictor of out-of-area placements and is a risk variable for abuse. Recent research suggests that mindfulness-based therapies (specifically, Singh's Soles of the Feet meditation) can help people with intellectual disabilities manage angry emotions, with resultant reductions in challenging behaviour. However, previous research has been single-case design studies, and no group studies have been published with people with intellectual disabilities and aggressive behaviour.

Methods/design: For this feasibility study, a UK protocol will be developed for use by health professionals within National Health Service (NHS) Intellectual Disability (ID) teams, based upon Singh's Soles of the Feet manual. Twenty adults with intellectual disabilities and identified problems with anger control will be recruited and six sessions will be delivered by a trained ID clinician. The study will monitor participant's aggressive behaviour, health-related quality of life, anxiety, depression, and use of support services (medication, hospital appointments etc.). These will be measured at three time points: (1) Baseline (within 2 weeks prior to the first session of the intervention), (2) 2 months post-baseline, and (3) 6 months post-baseline. Qualitative interviews will be conducted with participants, their carers, and the therapists who delivered the intervention. In order to help design an economic evaluation alongside a future full trial, we will cost the intervention and test the acceptability and validity of health economics measures to record resource use and health-related quality of life outcomes.

Discussion: The data from this study will inform the feasibility of the project protocol and intervention, which will help develop future research and to determine whether a larger, randomised controlled trial with concurrent economic evaluation is feasible.
\end{abstract}

Trial registration: UKCERN: 16743.

Keywords: Intellectual disability, Mindfulness-based, Aggressive behaviour, Feasibility study, UMAA-LD, Costs

\footnotetext{
* Correspondence: g.m.griffith@bangor.ac.uk

${ }^{1}$ Centre for Mindfulness Research and Practice (CMRP), Bangor University,

Bangor, UK

Full list of author information is available at the end of the article
} 


\section{Background}

In the UK, $2 \%$ of adults and $3.5 \%$ of children have an intellectual disability (ID) [1, 2]_defined as an IQ below 70 , alongside adaptive behaviour deficits, and onset within childhood. Approximately 10-20\% of people with ID engage in challenging behaviour [3-7], and challenging behaviours are likely to persist over extended periods of time [8]. Severe challenging behaviour has a range of negative impacts, such as a lower quality of life, a negative impact on carer well-being $[9,10]$, reduced access to community services, and admission to intensive and specialist, and therefore costly, residential, or forensic services [11].

When interviewed, some adults with ID report that they find their own anger and challenging behaviour difficult to cope with and aversive and described feeling regretful after engaging in challenging behaviour [12-14]. Qualitative studies and a recent meta-synthesis have found that adults with ID are keen to learn how to selfmanage anger [15].

Leading researchers on anger have called for creative and more effective interventions than the most common treatment methods such as cognitive behavioural therapy (CBT). CBT holds particular challenges for people with ID, as it requires high levels of language skills, and the ability to be introspective and self-aware, and yet does not explicitly address the development of such awareness [16].

There is an ongoing debate in the field about how best to define the complex construct of mindfulness (see [17]). An oft-cited definition of mindfulness is 'paying attention in a particular way, on purpose, in the present moment and non-judgementally ([18]. p.4)'. Mindfulness-Based Stress Reduction (MBSR) was originally developed in the late 1970s, and MBSR courses today closely follow the original format. MBSR courses are taught in groups, for around $2.5 \mathrm{~h}$ a week for 8 weeks, with a full day of silent meditation practice around the sixth week. Participants are given home practices of around $45 \mathrm{~min}$ to an hour each day [18].

Mindfulness has the potential to address a number of psychological processes and states that are relevant to the risk of anger and resultant challenging behaviour [16].

These include reduced emotional reactivity to a trigger by observing anger-related sensations in the body without attempting to avoid or act on them. Evidence suggests that mindfulness is particularly relevant to the reduction of negative emotional episodes [19] because rumination increases the emotion of anger [20]. Conversely, a nonjudgemental view of one's thoughts may help reduce the negative impact of ruminative patterns. The ability to selfmonitor one's own mood states is regarded as being central to anger regulation. To adjust an individual's anger response, there needs to be an awareness that anger is present. Aggressive outbursts may become autotomized anger responses, with little awareness of conscious attention (for people both with and without ID). Mindfulness training enables awareness of the bodily, cognitive, and behavioural signs of anger and opens the possibility of autotomizing non-aggressive responses [16].

Mindfulness-based interventions (MBIs) are recommended by the National Institute for Health and Care Excellence (NICE) in the UK. Specifically, mindfulness-based cognitive therapy (MBCT) is recommended for recurrent depression [21], and Dialectical Behaviour Therapy for Borderline Personality Disorder [22]. Typical MBIs rely on complex language to reflect on the experience of meditation, which may be cognitively demanding for individuals with ID. Therefore, it is important to consider how to adjust approaches for this population. In the USA, Soles of the Feet (SoF) meditation has been developed as a way of making MBIs accessible for people with ID [23]. It has been successfully used in ID populations to self-manage anger and, at long-term follow-up, has resulted in reported reductions or total elimination of aggressive behaviours [24-28]. It has also been used to help reduce deviant sexual arousal [29], smoking [30], and health behaviours contributing to obesity [31].

A recent systematic review [32] found the most frequent objective of MBIs for individuals with ID was the reduction of aggressive behaviour (explored in 7/12 studies), and of these, all reported a reduction in aggressive behaviour. Although there were 12 studies in the Hwang and Kearney review, this represents just 22 participants as they were all single-case design studies.

The Singh SoF manual [23] was originally designed to help individuals with ID cope with anger and reduce aggressive behaviour [25]. Individuals are taught to recognise situations that are 'triggers' for their anger, then guided through the steps of the SoF meditation. These include breathing naturally without trying to respond to angry thoughts or emotions and then shifting attention to, and being mindful of, the soles of their feet (a neutral part of the body). This technique is tailored to the cognitive level of the individual and enables the regulation of emotional states and behaviours. Thus, it is a good match with current philosophical and policy perspectives on empowering the lives of people with ID [32-34].

Hwang and Kearney [32] noted that teaching SoF to people with ID (in the studies, they reviewed) was labourintensive, which creates a problem with intervention accessibility for patients and providers alike. The authors called for group evaluation: "Diversifying intervention methods and research methodology is the key" (p. 325). The proposed study addresses both the issue of labour intensity and of group evaluation. First, we aim to develop an adapted SoF package: Using Mindfulness for Anger and Aggressive Behaviour with people with Learning Disabilities-Soles of the Feet (henceforth referred to as UMAA-LD SoF), that is 
delivered over six sessions by health professionals working in the UK National Health Service (NHS). This serves to create an intervention that is comparable or shorter in timescale to other developed clinical interventions for anger and aggressive behaviour in this population [35-38]. Second, we are utilising a group design and so taking a step up the 'evidence hierarchy' from the single-case designs used thus far with SoF with people with ID and aggressive behaviour. Third, it is important that researchers are able to measure levels of mindfulness within a population where intervention studies are being conducted. Existing measures of mindfulness such as the Five Facet Mindfulness Questionnaire [39] are likely to be too cognitively demanding for people with ID. Therefore, development and validation of a measure of mindfulness for this population will be undertaken as part of the UMAA-LD study.

Few studies evaluating MBIs consider the costs along with the possible clinical benefits [40]. In the original SoF evaluation [28], a benefit-cost analysis indicated significant cost savings following SoF delivery due to a reduction in staff absenteeism at work. The SoF intervention costs were not included in the analysis as they were absorbed into routine behaviour training costs. These savings may not be representative of the costs of delivery within a UK health care setting. In order to evaluate whether SoF is a cost-effective intervention, a full economic evaluation alongside a randomised controlled trial is necessary. We chose to conduct a feasibility study because we wanted to explore the practicality of the proposed intervention and methodology. Therefore, this feasibility study will contribute to the growing evidence base of using SoF for people with ID and anger and aggressive behaviour.

\section{Aims}

The principal aims of this feasibility study are

1) To test the UMAA-LD SoF protocol and research process including delivery across a NHS site, referral pathways, number of eligible participants and carers, treatment fidelity, willingness of clinicians to recruit, questionnaire completion rates, the time taken to complete outcome and process measures, and acceptability and accessibility of the UMAA-LD SoF protocol

2) To develop and carry out a preliminary test of the reliability and validity of a Mindful Awareness for adults with an Intellectual Disability Scale (MAIDS)

3) To cost the UMAA-LD SoF intervention and evaluate the acceptability and validity of the health economic outcomes for a subsequent full economic evaluation

4) To develop and test the feasibility of a protocol for a full-scale effectiveness randomised control trial (RCT) with concurrent economic evaluation of the UMAA-LD SoF intervention

\section{Methods \\ Design}

A single-arm study with 20 participants at three time points of measurement: baseline, immediately after intervention ( 2 months post-baseline), and follow-up (6 months post-baseline). A sample size of 20 is feasible and economical whilst providing sufficiently precise estimates of variances for powering larger studies. The UMAA-LD SoF intervention will take place over six sessions, at a rate of one session per week.

A researcher will visit participants at all three time points to assist with completion of outcome measures. This will take place either in the participant's home or a place of their choice (e.g. their local clinic).

\section{Participants}

Twenty participants will be recruited for the study. Inclusion criteria are (1) over the age of 18 years, (2) with an ID (established through the administration of the Wechsler Abbreviated Scale of Intelligence 2nd Edition [41]) and Adaptive Behaviour Assessment System $\odot$ (ABAS; $2^{\text {nd }}$ Edition [42]), (3) have clinically significant difficulty with anger control as assessed by their clinician, (4) able to give informed consent, and (5) has a family member or paid carer who has supported them for a minimum of 6 months, is available to participate in the treatment sessions, and who currently provides a minimum of 2-h support per week to the participant. $\mathrm{Al}$ though language ability is not an inclusion criterion, if participants are able to give informed consent, then they are also likely to have the language level needed to participate in an UMAA-LD SoF intervention.

Exclusion criteria are (1) existing diagnosis of autism spectrum disorder (because of potential difficulties with more abstract concepts relating to mindfulness when ID and autism are co-morbid), (2) presence of mental health problems or behaviour that may prevent the participant from interacting with the carer or therapist or retaining information from the therapy (e.g., dementia, active psychosis), and (3) individuals who are currently receiving direct psychological intervention (e.g. relaxation training, DBT, CBT).

\section{Recruitment}

The research team will meet with clinicians working in NHS ID teams prior to commencement and explain the study. If clinicians are willing to be involved in recruitment, they will be given a supply of study information packs, comprising a letter of invitation, a participant information sheet, a carer information sheet, a reply slip, and a Freepost return envelope addressed to the researcher. Clinicians will be required to explain to the person with ID that a follow-up telephone call can be made by the researcher should they agree to this. 
If the person with ID agrees to a follow up telephone call, clinicians will contact the potential participant once by telephone to check if they have retained the study information pack. If the pack has been mislaid, the clinician will offer to send a second pack. Adults with ID are often supported by several different carers, working across several settings (e.g. a person's home environment, day centre). This situation means there is a high likelihood of study packs going missing.

On receipt of reply slips with the name and contact details of the potential participant agreeing to contact by the researcher, the researcher will arrange an initial screening visit so that they can explain verbally to the person with ID and their carer what the study involves and so that informed consent can be sought. Further visits/telephone contact can be arranged should the potential participant require more time before making a decision.

\section{Procedure}

An initial screening visit (by a trained researcher) will seek informed consent and confirm whether the participant meets the inclusion criteria. If the inclusion criteria are met, the timescale for the three time points of measurement will commence. (1) Baseline (within 2 weeks prior to the first session of the intervention), (2) 2 months postbaseline, and (3) 6 months post-baseline. Data will be collected during face-to-face sessions with the researcher. The carer will also be available to support the person with ID should this be requested/necessary. At the 6-month postbaseline visit, the researcher will conduct semi-structured one-to-one interviews with participants and carers who took part in the intervention.

\section{Soles of the Feet meditation: intervention Development of materials}

The research team consists of international experts in the field of MBIs (RC) and intellectual disabilities (RJ, $\mathrm{RH}, \mathrm{JW})$ who will collaborate on the development of all materials. One of the researchers (JW) has experience of using variations of the original SoF protocol in routine clinical practice in the NHS prior to the trial. JW's prior clinical experience of using SoF mindfulness approaches with people with ID in community settings helped inform the development of the UMAA-LD SoF protocol, e.g. highlighting concepts which participants often found difficult, with suggestions about how to make these more accessible.

\section{Development of the UMAA-LD SoF protocol}

The core SoF meditations [23] will be used in the UMAA-LD SoF manual. Singh et al. [23] provides a detailed description of how to teach Soles of the Feet mediation in the original manual. There are three main SoF meditations, all follow the same broad therapist instructions but with a different emphasis on the evoked emotion. The steps outlined below demonstrate typical therapist guidance during a SoF meditation (step 2: adapted from [23]):

1. Sit or stand in a natural position with the soles of the feet flat on the floor.

2. Breathe naturally.

3. Think about an incident that made you feel angry.

4. Notice the angry thoughts and stay with them, just notice how your body feels, and any thoughts or emotions that are around.

5. Quickly shift the focus of your attention to the soles of your feet.

6. Now, wiggle your toes. Feel your socks on the soles of your feet, focus on the arches of your feet. Go to the heels of your feet.

7. Continue wiggling your toes.

8. Now, open your eyes.

9. Well done for doing this Soles of the Feet practice.

There are three SoF meditations, each one introduced to the participant in a particular order, each meditation is intended be repeated until the participant is able to follow the instructions with fluency. In published research, the training times for SoF were usually in two stages, the first, an intensive training period with a therapist, usually between 5 days and a week, followed by a longer period of self-practice (between 12 weeks and 2 years), often with an audio recording [32].

SoF meditation (1) Teaching Soles of the Feet meditation begins with the therapist asking the client to think of a happy situation and then following the steps of the meditation (see above).

SoF meditation (2) The participant then practises the same meditation, but this time whilst evoking a situation that make them feel angry.

SoF meditation (3) The participant then practises the meditation whilst thinking about a 'trigger' situation that typically causes them to get angry. This is the ultimate aim of the intervention-that the participant is able to identify triggering situations and use Soles of the Feet meditation appropriately in situ, before they get angry.

Although the original, core SoF practices [23] will be used, there will also be adaptions to the current manual, the main difference is that psycho-educational components around anger and stress reactions in the body will be added. The UMAA-LD SoF protocol will be designed as an addendum to the existing manual, rather than a replacement for it. The main additions will be as follows: 
1) UMAA-LD SoF will be designed specifically for use in NHS settings. The SoF manual [23] does not specify what content is covered on a session-by-session basis. The UMAA-LD SoF manual will provide specific outlines for each of six sessions. Each session will take up to $90 \mathrm{~min}$, with the clinician pacing the session to meet participants' needs. See Table 1 for a typical session format.

2) UMAA-LD SoF will provide educational materials suitable for people with ID that will focus on anger, aggression, and mindfulness. These will be used in sessions and also form a 'pack' that the participant can take home and read.

3) UMAA-LD SoF sessions will require the participant (with carer support) to engage in short, daily home meditation practices each week. The home practices given will follow the progression of the session. A $\mathrm{CD}$ /audio file that will have a recording of the Soles of the Feet meditation will be provided in a format best suited to the participant. These audio recordings of SoF practices will be specially designed for the ID population and will last for around 4-6 min each.

\section{Therapist training and supervision to deliver UMAA-LD SoF}

All therapists will be trained clinical psychologists, supervised trainee psychologists, intellectual disability nurses or other NHS clinicians. The UMAA-LD SoF training was for a full day, co-delivered by clinicians (RJ and JW), and a mindfulness-based teacher (GG). During the training day, the manual was explained on a session-by-session basis and core SoF practices were demonstrated and explained, and therapists practiced delivering the SoF meditations in the training session. Therapists were encouraged to attend an MBSR class if they had not already done so and to sustain their personal mindfulness practice after the training and during delivery of SoF sessions. This is due to the importance of mindfulness being communicated through the therapists embodied practice during sessions. Therapists will receive two supervision sessions over the six sessions for each participant. At least one supervision session will be with a mindfulness-based teacher (GG) to discuss issues pertaining to the mindfulness elements of the

Table 1 Typical format for a UMAA-LD SoF session

\begin{tabular}{ll}
\hline Content of session & Approximate time frame (min) \\
\hline Introducing the session & $5-10$ \\
Review homework log & $5-10$ \\
$\begin{array}{l}\text { Psycho-educational component, i.e. } \\
\text { what is anger? What is mindfulness? }\end{array}$ & $20-30$ \\
$\begin{array}{l}\text { Comfort break } \\
\text { Soles of the Feet meditation practice }\end{array}$ & $20-10$ \\
$\begin{array}{l}\text { Review of the session and home } \\
\text { practice setting for next week }\end{array}$ & $5-10$ \\
\hline
\end{tabular}

intervention. The other supervision session will be provided by a senior ID clinician (RJ or JW) or by a clinician and researcher (JR).

\section{Mindfulness scale for people with ID}

To address aim 2, the following methodology will be adhered to.

The MAIDS will be a self-report measure to elucidate mindfulness processes in relation to problem areas, such as anger. Development of items will be informed by three sources: (1) examination of existing mindfulness measures (no current measure exists for use with people with ID), (2) research on the development of self-report measures for people with ID, and (3) consultation with mindfulness and ID professionals. To date, a working version of MAIDS (consisting of ten items) has been developed. The MAIDS will be incorporated in the UMAA-LD SoF protocol to test its feasibility as an evaluation tool.

The MAIDS will be tested on the 20 participants in the intervention study, at all three time points. In addition, to help determine reliability and validity of the scale, 80 further participants with ID will be recruited to complete the MAIDS, the Modified Overt Aggression scale [43], Novoco Anger Scale [44], Glasgow Anxiety [45] and Glasgow Depression Scale [46] at a single time point. Participants will be recruited from various sources, including local ID groups and residential homes. Informed consent will be gained prior to collecting data and will be collected by researchers visiting the participants to assist where required.

\section{Measures}

A researcher is responsible for collecting all feasibility data and outcome measures. The primary aim of these outcome measures is to test the suitability and practicality of the measures with adults with ID. Following the initial screening visit, where capacity to consent is assessed, a baseline visit will be arranged prior to the commencement of the intervention where demographic information on age, gender, and current residential status will be gathered. All of the feasibility outcome measures described below will be taken at the three time points of baseline, 2 months post-baseline, and 6 months post-baseline.

\section{Feasibility process measures}

To address aim 1, the following measures will be taken.

1. Screening and recruitment rates will be monitored.

2. The MAIDS to measure skills in using mindfulness to manage anger.

3. Data on adherence to mindfulness home practice.

4. Participant drop out from the feasibility study will be closely monitored and reasons gathered where possible. 
5. An assessment of the completion rates of the questionnaires will be completed. This will encompass an analysis of any missing data patterns.

6. Information gleaned from the qualitative interviews will be used to inform understanding of the process.

7. Intervention fidelity will be monitored. Two out of six sessions with each participant will be audio recorded. One will be from the start of the intervention (sessions 1-3) and the other from sessions 4-6. The recordings will be evaluated using an adaptation of Singh's Soles of the Feet monitoring form that evaluates adherence to the Soles of the Feet manual [23].

8. A clinical input questionnaire will capture the participants' recent clinical input (e.g. from a behavioural specialist, any other direct psychological interventions) and be completed by the carer with the researcher during the consent visit, this is repeated during the baseline visit to identify any recent changes.

\section{Demographic measures}

Following the initial screening visit, where capacity to consent is assessed, a baseline visit will be arranged prior to the commencement of the intervention where demographic information on age, gender, and current residential status will be gathered. As part of the inclusion criteria, the presence of an ID will be confirmed at baseline using two measures: (1) Wechsler Abbreviated Scale of Interlligence-Second Edition [41] that measures IQ in adults and (2) Adaptive Behaviour Assessment System ${ }^{\circ}$ (ABAS) - Second Edition [42] that is completed by the carer and measures abilities, skills and physical, and sensory impairments.

\section{Measures (administered to the participant with ID)}

All of the potential outcome measures described below will be taken at the three time points of baseline, 2 months post-baseline, and 6 months post-baseline.

All self-report measures used have been adapted for use with an ID population or have been evaluated by the research team and are likely to be appropriate measures for use with individuals with ID.

Novaco Anger Scale (NAS): A 60-item scale exploring how an individual experiences anger, with three subscales: cognitive, arousal, and behavioural, that constitute a total score for anger disposition [44].

Provocation Inventory (PI): A 25-item scale that identifies the kinds of situations that induce anger in particular individuals [47].

The Glasgow Depression Scale for adults with Learning Disabilities (GDS-LD): A 20-item scale that asks how often depressive symptoms have occurred over the past week [46].
The Glasgow Anxiety Scale for adults with Intellectual Disabilities (GAS-ID): A 27-item self-rating scale that assesses how often anxiety symptoms have occurred over the past week [45].

EQ-5D-Y (Youth version of the EQ-5D generic Health Related Quality of Life questionnaire: HRQoL): This is a standardised and validated instrument on HRQoL and is intended for use with children and adolescents aged between 7 and 12 years old [48, 49]. There are currently no generic quality of life questionnaires adapted for people with ID.

CHU-9D (A paediatric generic preference based measure of $H R Q o L)$ : This is intended for use with children aged 7-17 years [50]. There are currently no generic quality of life questionnaires adapted for people with ID and challenging behaviour.

ICECAP-A (ICEpop CAPability measure for Adults): A measure of capability for the general adult (18+) population for use in economic evaluation [51].

\section{Potential outcome measures-proxy (carer) report}

The primary outcome measure is the Modified Overt Aggression Scale (MOAS: [43]) measures four types of aggressive challenging behaviour over the past week (verbal, against objects, against self, against others) and measures both severity and frequency. The MOAS has good internal consistency and test-retest reliability $(\alpha=0.75)$ for the rating of aggression. The MOAS will also be administered to the carer by the clinician delivering the intervention on a weekly basis.

Carers will also complete proxy versions of the EQ5D-Y [48], CHU-9D [50], and ICECAP-A [51] so that their perception of the quality of life of the person they care for can be measured. In addition, the following measures will be utilised.

The Glasgow Depression Scale-Carer supplement (GDS$C S)$ : A 16-item scale that asks how often depression symptoms have occurred over the past week [46]. Anxiety, Depression, and Mood Scale (ADAMS): As there is no equivalent of the GDS-CS41 for anxiety, the general anxiety scale of the ADAMS will be utilised as a proxy report measure of anxiety [52].

Client Service Receipt Inventory-European Version (CSRI-EU): This has been used in previous ID research and examines the participant's use of medication and health care, social care, day, and community services [53].

\section{Qualitative interviews}

Qualitative semi-structured one-to-one interviews with participants and carers will be conducted after all outcome data have been collected (6 months after baseline) focusing on the interview topics described below. 
Participants: How accessible/acceptable SoF meditation was, the impact upon their feelings and expressions of anger, their quality of life, adherence and perceived barriers to the intervention, use of the mindfulness practice audiofiles at home, whether they will continue to use SoF meditation, exploration of any relationship changes with their carer, and any future support needed.

Carers: Perceived impact of the intervention on their offspring/client, their relationship with their client/ family member throughout the intervention, and exploring what, if any, impact it had on their own lives, both personally and as a carer.

UMAA-LD SoF therapists: their experiences of mindfulness prior to training, their experience of being trained to use UMAA-LD SoF, experiences of delivering the UMAA-LD SoF, including the accessibility of the manual for themselves and clients and the perceived effects on participants and carers.

\section{Method of analysis}

As this is a feasibility study, no hypothesis is to be tested, and no formal analysis of outcome variables will be made as the study is not powered for definitive analysis.

The primary measures of interest are patient recruitment, attrition, and response rate for questionnaires. Feasibility metrics (e.g. recruitment and retention rates, clinical characteristics, duration of the intervention, etc.) will be analysed first, together with adherence outcomes (patient acceptance and adherence to the intervention). The mean change from baseline together with associated variances will be calculated for all measures and will be presented as point estimates together with $95 \%$ confidence intervals. An estimation of the precision of the means and variances will be made to inform the power calculation for the main RCT protocol. Framework analysis [54] is the planned approach to analysing the qualitative interview data.

Initial psychometric properties of the MAIDS will be evaluated using baseline data collection. Internal consistency for the MAIDS total score will be estimated in addition to initial convergent validity (association with baseline mental health measures).

\section{Health economic evaluation}

To address aim 3, the health economics component of the study will be conducted from a public sector multiagency perspective [55]. As there is limited evidence of self-reported HRQoL and service use questionnaires being used in an ID population, the principle aim of the health economics evaluation will involve piloting the key outcome measures. A comparison will be made between the participants' self-reported HRQoL and proxy ratings of HRQoL. Descriptive statistics will be compared with other study outcomes and available social norms. The correlation between study outcome measures will be assessed to explore the construct validity of measures in this population.

The intervention will be costed using micro-costing techniques that have previously been successfully used in costing MBIs and other complex psycho-social interventions [56]. Patterns of health care, social care, and other service use over the preceding four months will be explored and costed using national unit costs $[57,58]$. A period of 4 months is sufficient for a representative picture of service use to be gauged, yet recent enough for the respondent to recall accurately the frequency and nature of contacts $[59,60]$. As the intervention followup period is less than 1 year, it will not be necessary to discount costs [55].

\section{Protocol for a full-scale effectiveness trial}

To address aim 4, a full study would be considered feasible if (1) recruitment rate found was more than $50 \%$, (2) if the retention rate found was more than $50 \%$ at final follow-up, and (3) if adherence rate was greater than $50 \%$ for the completing participants.

\section{Ethics and consent}

Ethical approval has been received from both Bangor University, and the NHS Research Ethics Committee (ref $15 / \mathrm{WA} / 0213$ ), and the study is registered with the UK Clinical Research Network Study portfolio-database no. 16743. Our protocol around assessing capacity to consent has been designed in accordance with the Mental Capacity Act [61] and ID best practice [62]. We will use developmentally appropriate information sheets and consent forms. It will be made clear to participants that they have the right to withdraw from the study at any time. Those unable to consent and those who do not consent will continue to receive treatment as usual from their service. Consent will be sought from carers separately.

\section{Discussion}

The prevalence of aggressive behaviours among people with ID is further accentuated by limited access to evidencebased interventions. Leading ID researchers have called for creative and more effective interventions for anger [16].

Research in the field of ID shows the negative impact of challenging behaviour resulting from anger that can lead to a lower quality of life, a negative impact on carer well-being, reduced access to community services and admission to intensive and specialist, and therefore costly, residential, or forensic services [3,9-11]. Therefore, within specialist ID NHS services and mainstream mental health services, there is a need to further develop clinical practice to support adults with ID with mental health problems, 
including anger and aggressive behaviour. There has been some work to adapt and evaluate CBT methods for anger (although few examples of high-quality research), but little focus on adapting other psychological therapies from mainstream services.

This feasibility study will examine an evidence-based intervention for anger in adults with ID and is anticipated to benefit participants directly by offering a valuable treatment approach where limited approaches exist currently. An intervention informed by mindfulness may also have other benefits with an ID population because it encourages the individual to develop an attitude of shared responsibility for their own mental health with consistent practice of self-management skills. Thus, the proposed UMAA-LD SoF intervention is potentially useful both as an early intervention measure for reduction in aggressive behaviour and also as a strategy for longterm management of anger and aggressive behaviour. Mindfulness training is also non-intrusive, an alternative to medication and promotes resilience and well-being. By developing familiarity with mindfulness and basic meditation techniques, adults with ID may also be better placed to access mainstream mindfulness classes. Adults with ID may (perhaps with the support of their carer) be empowered to manage other emotional problems (e.g. anxiety) using their new transferable mindfulness skills. Finally, reducing anger and associated challenging behaviour may contribute towards participants' ability to engage with paid employment, improving their economic situation and providing meaningful activity.

In health economics evaluations, the NHS traditionally prefers a cost-utility approach to economic analysis, whilst in Public Health cost-benefit and costconsequence analysis are considered to be more appropriate by NICE [63]. The UMAA-LD SoF intervention may result in benefits and possible cost savings that goes beyond the health service; therefore, a public sector multi-agency approach to economic analysis is likely to capture this wider potential impact of the intervention.

There will likely be variability in therapists' previous experience of practicing or teaching mindfulness-based approaches. The UK Good Practice Guidelines for teachers of mindfulness-based programmes such as MBSR and MBCT stipulate that those delivering MBIs have personal experience of mindfulness practice [64]. However, due to practicalities of recruiting ID health professionals to deliver UMAA-LD SoF, we will aim for this but anticipate this will not always be possible. Those who do not have personal experience of mindfulness practice in advance will be offered support and encouragement to develop this. Although this intervention is mindfulness informed, it is not mindfulness-based, because it does not comply with UK good practice guidelines (although these were developed for teachers of the 8-week courses for the general public or clinical populations and are not intended to be specific for ID populations [64]). This aspect of the project will be closely monitored as part of the feasibility study, through the qualitative interviews with the therapists, as the personal practice of the teacher and how this is embodied could be important with an ID populations as it bypasses cognitive verbal channels.

We anticipate that the qualitative information will enhance the quantitative analysis and RCT protocol. Although preliminary data on the effectiveness of the intervention will be taken, it will not give insight into the mechanisms of change. This question will be explored in the qualitative data, with participants, their carers, and with clinicians. The qualitative data will also help identify any practical barriers or facilitators to participating in the intervention and any unanticipated positive or negative affects of the intervention.

Successful anger management intervention trials using RCT have been utilised for people with ID [38] allaying concerns expressed by some researchers about whether it is feasible to undertake RCT's with people with ID [65]. This feasibility study will examine whether UMAALD SoF may be suitable for investigation as a large-scale RCT. First, all data collected will be used to make any final revisions to the UMAA-LD SoF protocol, if necessary. Secondly, if the feasibility study is promising, an intervention package and protocol for an RCT with concurrent economic evaluation will be developed. The intervention package will include a validated UMAA-LD SoF manual, a new mindfulness measure with initial psychometric data (MAIDS), a complete description of the intervention, clinician training method, method to measure treatment fidelity, and follow-up procedures. The protocol will draw on pilot data for recruitment pathways and inclusion and exclusion criteria. It will provide valuable information into the feasibility of key issues such as recruitment and acceptability of the UMAA-LD SoF protocol with participants, carers, and clinicians.

\section{Acknowledgements \\ We would like to acknowledge Helen Davies for her role in revising the final manuscript. \\ Funding \\ This study has been funded by Health and Care Research Wales (Formally National Institute for Social Care and Health Research). The funding body was not involved in the design, data collection, analysis, or interpretation of the data.}

\section{Availability of data and materials}

Data and supporting materials are not available from this study at the time of this manuscript being submitted, as the materials have not been finalised, and all data has not been collected yet.

\section{Authors' contributions}

GG led the writing of this manuscript and was involved with the study design and the funding application. $\mathrm{RJ}$ is the principal investigator. $\mathrm{RJ}, \mathrm{RH}$, $J W$, and RC contributed to the study design and funding application. JR 
contributed to the study design and led on the ethics application. $\mathrm{ZH}$ contributed to the data analysis plan. RTE and LB were responsible for the health economics aspect of the study. All authors contributed to, read, and approved the final manuscript.

\section{Competing interests}

The authors declare that they have no competing interests.

\section{Consent for publication}

Not applicable.

\section{Ethics approval and consent to participate}

Ethical approval has been received from both Bangor University, and the NHS North Wales Research Ethics Committee (ref 15/WA/0213). All participants will give informed consent before being included in this study. Our protocol around assessing capacity to consent has been designed in accordance with the Mental Capacity Act and ID best practice.

\section{Author details}

${ }^{1}$ Centre for Mindfulness Research and Practice (CMRP), Bangor University, Bangor, UK. ${ }^{2}$ School of Psychology, Bangor University, Brigantia Building, Penrhalt Road, Bangor LL57 2AS, UK. ${ }^{3}$ Centre for Educational Development, Appraisal and Research: (CEDAR) University of Warwick, Coventry, UK. ${ }^{4}$ Denbighshire Complex Disabilities Team, North Wales, North Wales, UK. ${ }^{5}$ Centre for Health Economics and Medicines Evaluation (CHEME), Bangor University, Bangor, UK. ${ }^{6}$ North Wales Organisation for Randomised Trials in Health (NWORTH), Bangor University, Bangor, UK

Received: 9 March 2016 Accepted: 7 September 2016 Published online: 20 September 2016

\section{References}

1. Bittles AH, Petterson BA, Sullivan SG, Hussain R, Glasson EJ, Montgomery PD. The influence of intellectual disability on life expectancy. J Gerontol. 2002; $57: 470-2$.

2. Department of Health. Valuing people: a new strategy for learning disability for the 21st century - a White Paper. London: Stationary Office; 2001.

3. Allen DG, Lowe K, Moore K, Brophy S. Predictors, costs and characteristics of out of area placement for people with intellectual disability and challenging behavior. J Intellect Disabil Res. 2007:51:409-16.

4. Cooper SA, Smiley E, Jackson A, Finlayson J, Allan L, Mantry D, et al. Adults with intellectual disabilities: prevalence, incidence and remission of aggressive behaviour and related factors. J Intellect Disabil Res. 2009:53:217-32.

5. Jacobson JW. Problem behavior and psychiatric impairment within a developmentally disabled population. I: behavior frequency. Appl Res Ment Retard. 1982;3:121-39.

6. Kiernan C, Kiernan D. Challenging behavior in schools for pupils with severe learning difficulties. Men Han Res. 1994:7:177-201.

7. Oliver C, Murphy GH, Corbett JA. Self-injurious behaviour in people with mental handicap: a total population study. J Intellect Disabil Res. 1987;31:147-62.

8. Totsika V, Toogood S, Hastings RP, Lewis P. Persistence of challenging behaviours in adults with intellectual disability over a period of 11 years. J Intellect Disabil Res. 2008:52:446-57.

9. Hastings RP. Do challenging behaviors affect staff psychological well-being? Issues of causality and mechanism. Am J Men Ret. 2002a;107:455-467.

10. Hastings RP. Parental stress and behavior problems of children with developmental disability. J Int Dev Dis. 2002b;27:149-160.

11. Emerson E. Challenging behavior. Analysis and intervention in people with intellectual disabilities, 2nd ed. Cambridge: Cambridge University Press; 2000.

12. Brown J, Beail N. Self-harm among people with intellectual disabilities living in secure service provision: a qualitative exploration. J Appl Res Intellect Disabil. 2009;22:503-13.

13. Duperouzel H, Fish R. Hurting no-one else's body but your own: people with intellectual disability who self injure in a forensic service. J Appl Res Intellect Disabil. 2010;23:606-15.

14. Ruef MB, Turnbull AP, Turnbull HR, Poston D. Perspectives of five stakeholder groups: challenging behavior of individuals with mental retardation and/or autism. J Posit Behavinterv. 1999;1:43-58.

15. Griffith GM, Hutchinson L, Hastings RP. "I'm not a patient, I'm a person": the experiences of individuals with intellectual disabilities and challenging behavior - a thematic synthesis of qualitative studies. Clin Psychol Sci Pract. 2013:20:469-88.

16. Wright S, Day A, Howells K. Mindfulness and the treatment of anger problems. Aggress Violent Behav. 2009;14:396-401.

17. Grossman P, Van Dam NT. Mindfulness, by any other name...: trials and tribulations of sati in western psychology and science. Con Bud. 2011;12:219-39.

18. Kabat-Zinn J. Full catastrophe living: how to cope with stress, pain, and illness using mindfulness meditation. New York: Dell Publishing; 1991.

19. Teasdale JD. Metacognition, mindfulness and the modification of mood disorders. Clin Psychol Psychother. 1999;6:146-55.

20. Rusting $\mathrm{CL}$, Nolen-Hoeksema S. Regulating responses to anger: effects of rumination and distraction on angry mood. J Pers Soc Psychol. 1998;74:790.

21. National Institute for Health and Clinical Excellence. Depression: the treatment and management of depression in adults. 2009. http://www.nice.org.uk/nicemedia/pdf/CG90NICEguideline.pdf. Accessed Mar 2016.

22. National Institute for Health and Clinical Excellence. Borderline personality disorder; treatment and management. 2009. http://www.nice.org.uk/ nicemedia/pdf/CG78NICEGuideline.pdf. Accessed Mar 2016.

23. Singh NN, Singh J, Adkins A, Singh AN, Winton ASW. A trainer's manual for meditation on the Soles of the Feet: a mindful method of anger management. Virginia: One Publications; 2008.

24. Singh NN, Wahler RG, Adkins AD, Myers RE. Soles of the Feet: a mindfulnessbased self-control intervention for aggression by an individual with mild mental retardation and mental illness. Res Dev Disabil. 2003;24:158-69.

25. Singh NN, Lancioni GE, Winton ASW, Singh J, Singh ANA, Singh ADA. Peer with intellectual disabilities as a mindfulness based anger and aggression management therapist. Res Dev Dis. 2011a;32:2690-2696.

26. Adkins AD, Singh AN, Winton ASW, McKeegan GF, Singh J. Using a mindfulnessbased procedure in the community: translating research into practice. J Child Fam Stud. 2010;19:175-83.

27. Singh NN, Lancioni GE, Winton ASW, Adkins AD, Singh J, Singh AN. Mindfulness training assists individuals with moderate mental retardation to maintain their community placements. Behav Modif. 2007:31:800-14.

28. Singh NN, Lancioni GE, Winton ASW, Singh AN, Adkins AD, Singh J. Clinical and cost-benefit outcomes of teaching a mindfulness-based procedure to adult offenders with intellectual disabilities. Behav Modif. 2008;32:622-37.

29. Singh NN, Lancioni GE, Winton ASW, Singh AN, Adkins AD, Singh J. Can adult offenders with intellectual disabilities use mindfulness-based procedures to control their deviant sexual arousal? Psychol Crime Law. 2011:17:165-79.

30. Singh NN, Lancioni GE, Winton ASW, Singh ANA, Singh J, Singh ADA. Effects of a mindfulness-based smoking cessation program for an adult with mild intellectual disability. Res Dev Disabil. 2008:32:1180-5.

31. Singh NN, Lancioni GE, Singh ANA, Winton ASW, Singh ADA, Singh J. A mindfulness-based health wellness program for individuals with Prader-Willi syndrome. J Ment Health ResIntellect Disabil. 2011;4:90-106.

32. Hwang Y-S, Kearney P. A systematic review of mindfulness intervention for individuals with developmental disabilities: long-term practice and long lasting effects. Res Dev Disabil. 2003:34:314-26.

33. Welsh Assembly Government. Policy and practise for adults with a learning disability. 2007. http://wales.gov.uk/topics/health/socialcare/disability/ learning/. Accessed 12 Nov 2015

34. Welsh Assembly Government. Our healthy future. 2009. http:/wales.gov.uk/ docs/phhs/publications/100527technicalen.pdf. Accessed 12 Nov 2015.

35. Welsh Assembly Government. Learning Disability Strategy: section 7 guidance on service principles and service responses. 2004. http://www. Idiag.org.uk/documents/sp-response-guide-e.pdf Accessed 12 Nov 2015.

36. Taylor JL, Novaco RW, Gillmer B, Thorne I. Cognitive-behavioural treatment of anger intensity among offenders with intellectual disabilities. J Appl Res Intellect Disabil. 2002:15:151-65.

37. Taylor JL, Novaco RW, Gillmer BT, Robertson A, Thorne I. Individual cognitivebehavioural anger treatment for people with mild-borderline intellectual disabilities and histories of aggression: a controlled trial. Br J Clin Psychol. 2005:44:367-82.

38. Willner P, Rose J, Jahoda A, Kroese BS, Felce D, Cohen D, et al. Group-based cognitive-behavioural anger management for people with mild to moderate intellectual disabilities: cluster randomised controlled trial. Br J Psychiatry. 2013;203:288-96

39. Baer RA, Smith GT, Lykins E, Button D, Krietemeyer J, Sauer S, et al. Construct validity of the five facet mindfulness questionnaire in meditating and nonmeditating samples. Assessment. 2008;15:329-42. 
40. Edwards RT, Bryning L, Crane R. Design of economic evaluations of mindfulnessbased interventions: ten methodological questions of which to be mindful. Mind. 2015;6:490-500.

41. Wechsler D. Welsher Abbreviated Scale of Intelligence-2nd Edition (WASI-II). San Antonio: Pearson Assessment; 2011.

42. Harrison P, Oakland T. Adaptive Behaviour Assessment System -2nd Edition (ABAS-II). San Antonio: The Psychological Corporation; 2003.

43. Oliver PC, Crawford MJ, Rao B, Reece B, Tyrer P. Modified Overt Aggression Scale (MOAS) for people with intellectual disability and aggressive challenging behaviour: a reliability study. J Appl Res Intellect Disabil. 2007;20:368-72.

44. Novaco RW. The Novaco Anger Scale and Provocation Inventory (NAS-PI). Los Angeles: Western Psychological Services; 2003.

45. Mindham J, Espie CA. Glasgow Anxiety Scale for people with an Intellectual Disability (GAS-ID): development and psychometric properties of a new measure for use with people with mild intellectual disability. J Intellect Disabil Res. 2003;47:22-30.

46. Cuthill FM, Espie CA, Cooper SA. Development and psychometric properties of the Glasgow Depression Scale for people with a Learning Disability. Individual and carer supplement versions. Br J Psychiatry. 2003;182:347-53.

47. Novaco RW, Taylor JL. Assessment of anger and aggression in male offenders with developmental disabilities. Psychol Assess. 2004;16:42-50.

48. EuroQoL group. EuroQoL-a new facility for the measurement of healthrelated quality of life. Health Policy. 1990;16:199-208.

49. Wille N, Badia X, Bonsel G, Burstrom K, Cavrini G, Devlin N, et al. Development of the EQ-5D-Y: a child-friendly version of the EQ-5D. Qual Life Res. 2010;19:875-86.

50. Stevens K. Developing a descriptive system for a new preference-based measure of health-related quality of life for children. Qual Life Res. 2009;18:1105-13.

51. Al-Janabi H, Flynn T, Coast J. Development of a self-report measure of capability wellbeing for adults: the ICECAP-A. Qual Life Res. 2012;21:167-76.

52. Ebensen AJ, Rojahn J, Aman MG, Ruedrich S. Reliability and validity of an assessment instrument for anxiety, depression, and mood among individuals with mental retardation. J Autism Dev Disord. 2003;33:617-29.

53. Chisholm D, Knapp MRJ, Knudsen HC, Amaddeo F, Gaite L, Van Wijngaarden B. Client Socio-Demographic and Service Receipt Inventory-European Version: development of an instrument for international research. Br J Psychiatry. 2000;177:28-33.

54. Srivastava A, Thomson SB. Framework analysis: a qualitative methodology for applied policy research. JOAAG. 2009:4:72-9.

55. Drummond MF, Sculpher MJ, Claxton K, Stoddart GL, Torrance GW. Methods for the evaluation of health care programmes. 4th ed. UK: Oxford University Press; 2015.

56. Charles JM, Edwards RT, Bywater T, Hutchings J. Micro-costing in public health economics: steps towards a standardised framework, using the Incredible Years Toddler Parenting Programme as a worked example. Prev Sci. 2013;14:377-89.

57. Curtis L. Unit Costs of Health and Social Care 2013. Personal social services research unit. 2013. http://www.pssru.ac.uk/project-pages/unit-costs/2013/. Accessed 12 Nov 2015

58. Department of Health. Reference costs 2013-2014. 2014. https://www.gov. uk/government/uploads/system/uploads/attachment_data/file/380322/01_ Final_2013-14_Reference_Costs_publication_v2.pdf. Accessed 12 Nov 2015.

59. Roberts RO, Bergstralh EJ, Schmidt L, Jacobsen SJ. Comparison of selfreported and medical record health care utilization measures. I Clin Epidemiol. 1996:49:989-95.

60. Ritter PL, Stewart AL, Kaymaz H, Sobel DS, Block DA, Lorig KR. Self-reports of health care utilization compared to provider records. J Clin Epidemiol. 2001;54:136-41.

61. Department of Health. Mental Capacity Act. London: HMSO; 2005.

62. Arscott K, Dagnan D, Kroese BS. Consent to psychological research by people with an intellectual disability. J Appl Res Intellect Disabil. 1998;11:77-83.

63. NICE. Methods for the development of NICE public health guidance. [PMG4]. 2012. http://www.nice.org.uk/aboutnice/howwework/developing nicepublichealthguidance/publichealthguidanceprocessandmethodguides/ public_health_guidance_process_and_method_guides.jsp. Accessed 12 Nov 2015

64. UK Network for Mindfulness-Based Teacher Training Organisations. Good practice guidelines of teaching mindfulness-based courses. 2015. http:// www.mindfulnessteachersuk.org.uk/\#guidelines. Accessed 12 Nov 2015.

65. Oliver PC, Piachaud J, Done J, Regan A, Cooray S, Tyrer P. Difficulties in conducting a randomized controlled trial of health service interventions in intellectual disability: implications for evidence-based practice. J Intellect Disabil Res. 2002;46:340-5.

\section{Submit your next manuscript to BioMed Central and we will help you at every step:}

- We accept pre-submission inquiries

- Our selector tool helps you to find the most relevant journal

- We provide round the clock customer support

- Convenient online submission

- Thorough peer review

- Inclusion in PubMed and all major indexing services

- Maximum visibility for your research

Submit your manuscript at www.biomedcentral.com/submit 